\title{
Thermal/pH-sensitive core-shell copolymer latex and its potential for targeting drug carrier application
}

\author{
Chia-Lung Lin, Wen-Yen Chiu*, Chia-Fen Lee \\ Department of Materials Science and Engineering, National Taiwan University, Taipei, Taiwan, ROC
}

Received 4 April 2005; received in revised form 28 June 2005; accepted 31 July 2005

Available online 19 August 2005

\begin{abstract}
In this work, the synthesis, properties of a thermal/pH-sensitive core-shell copolymer latex were studied and its potential application as a targeting drug carrier was also investigated, where the crosslinked copolymer of $N$-isopropylacrylamide (NIPAAm) and chitosan was synthesized by soapless dispersion polymerization as the core, and the copolymer of methacrylic acid (MAA) and methyl methacrylate (MMA) was prepared as the shell. The weight ratio of chitosan/NIPAAm and the concentration of crosslinking agent in feed had been changed to investigate their effects on the particle size, reaction rate, zeta potential, surface functional groups, and specific surface area of latex particles. The swelling and thermo-sensitive behavior of the film made from these core-shell latexes were also studied under different $\mathrm{pH}$ values of buffer solution. The model drug (caffeine) could be loaded inside the copolymer particles and protected from releasing through the transport process effectively. And the thermo-responsive property of these copolymer particles significantly enhanced the protein conjugation that showed the potential of the latex being applied on the targeting drug carrier.
\end{abstract}

(C) 2005 Elsevier Ltd. All rights reserved.

\section{Introduction}

Poly( $N$-isopropylacrylamide) (polyNIPAAm) has the behavior of temperature-responsiveness, which has been well documented [1-3]. Aqueous solutions of this polymer exhibit a lower critical solution temperature (LCST) at $32{ }^{\circ} \mathrm{C}$ [1]. Recently, new NIPAAm derivatives were designed with the goals of effectively controlling the temperature of phase transition by adding some hydrophilic or hydrophobic comonomers. Additionally, some new stimuli responsive properties were also developed in NIPAAm derivatives around the critical temperature [4-9]. Several authors have studied the behavior of aqueous colloidal dispersions formed from polyNIPAAm [10-16]. Makino et al. [16] prepared the core-shell particles; the

\footnotetext{
* Corresponding author. Tel.: +8862 23623259; fax: +886223623259.

E-mail address: ycchiu@ccms.ntu.edu.tw (W.-Y. Chiu).
}

0032-3861/\$ - see front matter (C) 2005 Elsevier Ltd. All rights reserved. doi:10.1016/j.polymer.2005.07.098 polystyrene (PS) core was coated with a polyNIPAAm network by radical polymerization. Napper et al. [17,18] synthesized polyNIPAAm, which grafted with PS. This graft copolymer was then used to stabilize a PS soap-free emulsion polymerization.

In addition, polyNIPAAm has been used widely as the material of drug delivery systems. A self-regulated drug delivery system has been of great interest because it allows the drug to be released when it is needed [4-6,19-23]. Park [6] developed a matrix-type delivery system for temperature-regulated release from a hydrogel system, wherein insulin crystals were entrapped within a $\mathrm{pH} /$ temperaturesensitive hydrogel matrix. A $\mathrm{pH} /$ temperature-sensitive hydrogel composed of NIPAAm and $N, N^{\prime}$-diethylaminopropyl methacrylamide, which exhibited a $\mathrm{pH}$-sensitivity around $\mathrm{pH} 7.4$ and a temperature-sensitivity around $37^{\circ} \mathrm{C}$, was synthesized. Park reported that temperature and thermal cycling operations largely affected the insulin release rate, while $\mathrm{pH}$ variation did not significantly influence the release profiles. Kono et al. [24] designed liposomes coated with a copolymer of NIPAAm and $N, N^{\prime}$-didodecylacrylamide. The 
release of calcein from the copolymer-modified liposomes was very slow below the phase transition temperature (LCST) of the copolymer, whereas the release was rapid above the phase transition temperature. The incorporation of dioleoylphosphatidylethanolamine into liposome membranes greatly enhanced release above the phase transition temperature of the copolymer.

Wang et al. [25] investigated the properties of chitosan (CS) and CS-polyNIPAAm hydrogels, which were prepared using glutaraldehyde (Glu) as a crosslinker. They pointed out that CS/Glu and polyNIPAAm-containing CS/Glu gel exhibited similar properties in all aspects examined, except that the transparence of the CS-polyNIPAAm/Glu gel was very dependent upon the temperature. Kim et al. [26] prepared the graft copolymer or blending of chitosan and polyNIPAAm. They pointed out that in the buffer solution of various $\mathrm{pH}$ values and temperatures, the chitosan/ polyNIPAAm blend IPN had a somewhat higher swelling than that of the poly (chitosan- $g$-NIPAAm) IPN. Wang et al. [27] studied the preparation and swelling behaviors of the chitosan/polyNIPAAm semi-IPN and IPN hydrogels. They found out that the properties of the gel, including the extractability of polyNIPAAm within it, the phase transition behavior, the swelling dynamics in aqueous phase, the swelling behavior in ethanol/water mixtures and even the microstructure were quite different from those of the semiIPN chitosan/polyNIPAAm hydrogels, in which polyNIPAAm was simply embedded [27].

In our previous study $[28,29]$, we designed the crosslinked poly(chitosan-co-NIPAAm) copolymer particles via soapless dispersion copolymerization [28-30] as a material of the drug delivery system in order to have the advantage of temperature sensitivity and biodegradability. But it was found that when the swollen poly(chitosan-coNIPAAm) copolymer particles were put into a $37{ }^{\circ} \mathrm{C}$ buffer solution from room temperature suddenly, the drug solution trapped in it would release quickly. Therefore, a novel coreshell type copolymer latex, poly(chitosan-co-NIPAAm/ MAA-co-MMA), was designed and synthesized in this study. The added poly(MAA-co-MMA) shell part could protect the drug from releasing through the transport process in human body and provide the carboxylic acid groups for the conjugation of ligand (protein). Therefore, these latices could be applied in the drug targeting. The residual reactants after the core synthesis, chitosan and NIPAAm, incorporated in the shell region and formed 'channels' which were expected to let the enzyme (lysozyme) pass through the shell to degrade chitosan in the core. Then the drug would be released on the targeted wounded tissue after the regulative degradation of copolymer particles. The effects of core composition variables, such as the weight ratio of chitosan/NIPAAm and the concentration of crosslinking agent on the reaction rate and the morphology of latex particles, were discussed. The swelling, thermo-sensitive and drug release behavior of these latex particles were also investigated.

\section{Experimental}

\subsection{Material}

$N$-Isopropylacrylamide (NIPAAm), methacrylic acid (MAA), methyl methacrylate (MMA), initiator (ammonium persulfate (APS)), crosslinking agent $\left(N, N^{\prime}\right.$-methylenebisacrylamide (MBA)) and model drug (caffeine) were all purchased from ACROS company and used as supplied. Chitosan was purchased from TCI company. Bovine Serum Albumin (BSA, Mw $\sim 66 \mathrm{kDa}$ ) and 1-ethyl-3-(3-dimethylaminopropyl) carbodiimide (EDC) were purchased from the Sigma Chemical Company and used as received. Water used in this experiment was redistilled and deionized.

\subsection{Soapless dispersion copolymerization}

The crosslinked poly(chitosan-co-NIPAAm/MAA-coMMA) core-shell copolymer latex was synthesized by the method of soapless dispersion copolymerization [28-30]. In the first stage of this copolymerization, the core latex, poly(chitosan-co-NIPAAm), was prepared as mentioned in our previous studies $[28,29]$ under the conditions listed in Table 1. After the first stage of synthesis was completed, the second stage of copolymerization was followed under the condition listed in Table 1. Finally, the core-shell latex particles with poly(chitosan-co-NIPAAm) as core and poly(MAA-co-MMA) as shell, were synthesized. Then the latex product underwent dialysis for 7 days in order to remove unreacted components. The sample code, for example $2 / 10-3 \%-1 / 5$, of the core-shell copolymer indicates the composition of feed mixture, where $2 / 10$ is the weight ratio of chitosan/NIPAAm, $3 \%$ is the concentration of crosslinking agent in MBA (g)/NIPAAm (g), and 1/5 is the weight ratio of MAA (g)/MMA (g).

\subsection{Conversion}

After the copolymerization, a quantitative amount of inhibitor was introduced into the latex and the sample was

Table 1

Ingredients and conditions for synthesis of crosslinked poly(chitosan-coNIPAAm/MAA-co-MMA) copolymer latex $\left(\mathrm{N}_{2} ; 50{ }^{\circ} \mathrm{C}\right.$; stirring rate, $300 \mathrm{rpm})$

\begin{tabular}{ll}
\hline First stage (core latex) & \\
NIPAAm (g) & 7 \\
Chitosan (g) & $1.4-3.5$ \\
MBA (crosslinking agent) (g) & $0.21-0.7$ \\
Initiator (APS) (g) & 0.7 \\
Acetic acid (g) & 1.5 \\
Deionized water (g) & 280 \\
Reaction time (h) & 2 \\
Second stage (core-shell latex) & \\
MAA (g) & 0.7 \\
MMA (g) & 3.5 \\
Reaction time (h) & 1 \\
\hline
\end{tabular}


cooled to room temperature for more than $1 \mathrm{~h}$, and then reheated to $60{ }^{\circ} \mathrm{C}$ (above the LCST of polyNIPAAm) to obtain the precipitates of the copolymers. The precipitates were washed with hot water several times for the removal of the remaining monomers and initiators, and then dried in a vacuum oven at $50{ }^{\circ} \mathrm{C}$ until the weight was no longer changed. The conversion of the monomers was calculated as follows.

Conversion $=\frac{P}{\left(W \times M_{0}\right)} \times 100 \%$

where $P$ is the weight of the dry copolymer obtained from the latex sample, $W$ is the weight of the latex sample taken out from the reactor, and $M_{0}$ is the weight fraction of monomers in feed.

\subsection{Morphology of copolymer particles}

To observe the morphology of the copolymer latex, the latex solution was coated on a glass plate and observed by TEOL JSM-6300 Scanning Electron Microscope (SEM). In addition, the samples were microtomed to form sections about 900A thick. The ultrathin cross-sections were stained with $1 \%$ uranyl acetate solution for $2 \mathrm{~min}$. Warm distilled water was used for the final rinse of the grid to avoid uranyl acetate precipitate. The stained ultrathin cross-sections were observed by using a transmission electron microscope (Hitachi H-600).

\subsection{Measurement of swelling ratio}

The latices were dried and modeled to form a polymer film in an oven at $50{ }^{\circ} \mathrm{C}$. Then it was immersed in an excess of deionized water for $30 \mathrm{~min}$ to remove the residual unreacted compounds and cut into disk forms of $8 \mathrm{~mm}$ in diameter, and then dried at $50{ }^{\circ} \mathrm{C}$ until the weight was no longer changed. A pre-weighed dried sample $\left(W_{\mathrm{d}}\right)$ was immersed in an excess of the $\mathrm{pH}=4, \mathrm{pH}=7.4$ and $\mathrm{pH}=9$ phosphate buffer solution in the thermostatic water bath until the swelling equilibrium was attained (i.e. the weight of the swollen film was no longer changed). The weight of wet sample $\left(W_{\mathrm{s}}\right)$ was determined after removing the surface water by blotting with filter paper. The equilibrium-swelling ratio (SR) was calculated from the following formula.

$\mathrm{SR}=\frac{\left(W_{\mathrm{s}}-W_{\mathrm{d}}\right)}{W_{\mathrm{d}}}$

\subsection{Zeta potential and light scattering measurements}

The zeta potential and hydrodynamic diameter (diameter of swollen copolymer particles) were measured using laser light scattering (Malvern Zeta Sizer 3000) at $25^{\circ} \mathrm{C}$ where the samples had been dialyzed for 7 days and diluted with phosphate buffer solution of $\mathrm{pH}=4, \mathrm{pH}=7.4$ and $\mathrm{pH}=9$.

\subsection{Specific surface area and surface functional group analysis}

The latex of copolymer particles was dialyzed for 7 days and freeze dried to get the powders to analyze the specific surface area and the amount of carboxylic acid groups on the surface of copolymer particles. The BET specific surface area of particles was measured and analyzed using Micromeritics ASAP-2100. A given quantity of the copolymer powder was dispersed in deionic water, followed by ultrasonification for $5 \mathrm{~min}$. The amount of carboxylic acid groups on the surface of copolymer particles was estimated by the $0.01 \mathrm{~N} \mathrm{NaOH}$ titration method using conductivity measurements.

\subsection{Caffeine loading into the copolymer}

Pre-weighed dried copolymer particles $(0.15 \mathrm{~g})$ were immersed in a $\mathrm{pH}=7.4$ phosphate buffer solution containing caffeine $(0.012 \mathrm{~g}$ caffeine $/ 5 \mathrm{~g}$ pH 7.4 phosphate buffer solution), followed by ultrasonification for $10 \mathrm{~min}$ and at the temperature of $4{ }^{\circ} \mathrm{C}$ for $3 \mathrm{~h}$ to attain the swelling equilibrium. Then these solutions were freeze dried to obtain the caffeine incorporated copolymer powder. $0.05 \mathrm{~g}$ of this powder was placed on a mold and pressed with 9 Tons of force to get the caffeine incorporated copolymer tablet with $1 \mathrm{~cm}$ diameter. The weight of the caffeine incorporated copolymer tablet was designated as $W_{\mathrm{c}}$. Therefore, the caffeine loaded into the tablet could be calculated as follows:

$L_{\mathrm{c}}$ (g caffeine loaded into tablet)

$$
=W_{\mathrm{c}} \times \frac{0.012}{0.15+0.012}
$$

The caffeine incorporated dry copolymer tablet was immersed into a phosphate buffer solution $\left(100 \mathrm{~g}, 37^{\circ} \mathrm{C}, \mathrm{pH}\right.$ 7.4) to release the caffeine. The quantity of caffeine released from each sample into buffer solution $\left(R_{\mathrm{c}}\right)$ was measured using a UV-vis spectrophotometer (Thermo Spectronic gamma series) by detecting the characteristic absorbance of caffeine at $272 \mathrm{~nm}$. The release percent of caffeine was determined as follows:

Release percent $(\%)=\frac{R_{\mathrm{c}}}{L_{\mathrm{c}}} \times 100 \%$

\subsection{Protein conjugation}

Pre-weighed dried copolymer particles $(0.01 \mathrm{~g})$ were immersed in a $\mathrm{pH} 7.4$ phosphate buffer solution $(10 \mathrm{~g})$, followed by ultrasonification for $10 \mathrm{~min}$ to suspend the copolymer particles in the solution homogeneously. Then, $0.004 \mathrm{~g}$ BSA was added into the solution and followed by re-ultrasonification for $3 \mathrm{~min}$. After the solution was placed in a 25 or $37^{\circ} \mathrm{C}$ thermostatic environment for $2 \mathrm{~h}, 0.004 \mathrm{~g}$ 
EDC was added into the solution for $4 \mathrm{~h}$ to conjugate BSA on the surface of copolymer particles. For estimating the amount of BSA conjugated on the surface of copolymer particles, the solution was centrifuged for $20 \mathrm{~min}$ at 12 , $000 \mathrm{rpm}$. The quantities of BSA remaining in the solution, which were not conjugated on the particles, was measured using UV-vis spectrophotometer (Thermo Spectronic gamma series) by detecting the characteristic absorbance of BSA at $280 \mathrm{~nm}$.

\section{Results and discussion}

\subsection{Preparation of copolymer latex particles}

The synthesis of the core-shell copolymer particles was conducted by a two-stage process.

\subsubsection{First stage of copolymerization}

In the first stage, the poly(chitosan-co-NIPAAm) core particles were prepared by soapless dispersion polymerization for $2 \mathrm{~h}$. The synthesis mechanism of the crosslinked poly(chitosan-co-NIPAAm) copolymer latex was discussed in our previous work [28]. Thermal decomposition of the initiator $\left(\mathrm{S}_{2} \mathrm{O}_{8}^{2-}\right)$ initiated the free-radical polymerization of NIPAAm, and the backbone of chitosan molecule was broken by free-radicals [31]. Consequently, the copolymerization of NIPAAm and chitosan occurred. From the ultrathin cross-section observation of the poly(chitosan-co-NIPAAm) copolymer particles stained by $\mathrm{RuO}_{4}$, it showed that chitosan distributed inside particles homogeneously [28].

Initially, NIPAAm was a water-soluble monomer, and oligomers of chitosan-co-NIPAAm formed in water. As the reaction proceeded, when the crosslinking degree achieved a certain level and the length of the oligomers exceeded the solubility limit, poly(chitosan-co-NIPAAm) precipitated out of the water phase and formed the primary particles. The primary particles underwent aggregation, thereby increasing the surface charge, until electrostatic stabilization was achieved. The particles grew with the aggregation and became more hydrophobic when polymerization was carried out at a temperature higher than LCST.

3.1.1.1. Effect of chitosan/NIPAAm weight ratio. The conversion data in Table 2 show that the increase of chitosan/NIPAAm weight ratio increased the conversion of copolymerization. The chitosan behaved as a surfactant, preventing polymer particles from coagulation [28], thereby increasing the particle concentration and the conversion of the polymerization. The average diameter of core particles decreased as increasing the weight ratio of chitosan/ NIPAAm, as expected. This was shown in our previous work [29].

3.1.1.2. Effect of crosslinking agent. By increasing the amount of crosslinking agent in the first stage
Table 2

Conversion of the NIPAAm/chitosan copolymerization (core latex) for $2 \mathrm{~h}$

\begin{tabular}{lll}
\hline $\begin{array}{l}\text { Chitosan/NIPAAm } \\
(\mathrm{w} / \mathrm{w})\end{array}$ & $\begin{array}{l}\text { Concentration of } \\
\text { crosslinking agent } \\
(\%)\end{array}$ & Conversion $(\%)$ \\
\hline $2 / 10$ & 3 & $82.02 \pm 0.15$ \\
$3 / 10$ & & $82.98 \pm 0.23$ \\
$5 / 10$ & & $85.91 \pm 0.22$ \\
$2 / 10$ & 3 & $82.02 \pm 0.15$ \\
& 5 & $81.25 \pm 0.19$ \\
& 10 & $81.02 \pm 0.13$ \\
\hline
\end{tabular}

copolymerization, conversion at $2 \mathrm{~h}$ was found to be slightly lowered as shown in Table 2 . Two effects were responsible for this result: (1) The crosslinking structure reduced the solubility of polyNIPAAm chains in water, and the polymers with a higher degree of crosslinking precipitated out of the water phase faster. Therefore, the increase in the concentration of the crosslinking agent increased the number of nuclei generated in the reaction system, reduced the particle size, and the polymerization rate could be enhanced. (2) The crosslinking structure hindered the diffusion rate of the monomer and, therefore, lowered the reaction rate of copolymerization. From the results in Table 2, effect (2) slightly dominated in our reaction system.

\subsubsection{Second stage of copolymerization}

After the first stage reaction, the monomers of MAA and MMA were added into the reaction latex to synthesize the shell part of particles. The second stage reaction was similar to the seeded emulsion polymerization, in which the poly(chitosan-co-NIPAAm) particles synthesized in the first stage were the seeds. The MAA and MMA, together with the unreacted chitosan and NIPAAm, were mostly adsorbed on the seeds and copolymerized to form the shell region of the particles. The chitosan and NIPAAm incorporated in the shell region would enhance the compatibility between core and shell region. In addition, they also formed channels in the rigid shell to absorb or release drug and to prevent the disappearance of thermoresponsive property when the rigid shell was expected to hider the swelling of the core region. These channels formed by chitosan and NIPAAm in the shell region were also expected to let enzyme pass through the shell to degrade chitosan in the core when the endocytosis was caused by the ligand on the particle surface.

3.1.2.1. Effect of chitosan/NIPAAm weight ratio. From the conversion data in Table 3, the increase of chitosan/ NIPAAm weight ratio increased the conversion of the second stage copolymerization. This could be due to the fact that chitosan acted as a surfactant and the higher concentration of polymer seed particles accelerated the copolymerization rate. 
Table 3

Conversion of the poly(chitosan-co-NIPAAm/MAA-co-MMA) core-shell copolymerization for $1 \mathrm{~h}$ after MAA/MMA was fed into different core latexes listed in Table 2

\begin{tabular}{lll}
\hline Core type & MAA/MMA (w/w) & Conversion $(\%)$ \\
\hline $2 / 10-3 \%$ & $1 / 5$ & $85.63 \pm 0.17$ \\
$3 / 10-3 \%$ & & $87.38 \pm 0.15$ \\
$5 / 10-3 \%$ & & $88.59 \pm 0.21$ \\
$2 / 10-3 \%$ & $1 / 5$ & $85.63 \pm 0.17$ \\
$2 / 10-5 \%$ & & $86.84 \pm 0.27$ \\
$2 / 10-10 \%$ & & $88.42 \pm 0.16$ \\
\hline
\end{tabular}

3.1.2.2. Effect of crosslinking agent. From the conversion data in Table 3, it was also observed that the increase of the crosslinking agent fed in the first stage reaction increased the conversion of the second stage copolymerization because the increase in the crosslinking agent would increase the hydrophobic property of the core particles, which functioned as effective seeds in the second stage copolymerization. In addition, the higher concentration of polymer core particles, which occurred when the concentration of crosslinking agent was higher, caused the polymerization rate to increase in the second stage polymerization.

\subsection{Observation of latex particles}

Fig. 1(a) shows the SEM photograph of the poly (chitosan-co-NIPAAm) core particles in the first stage copolymerization, which exhibited higher hydrophilic and
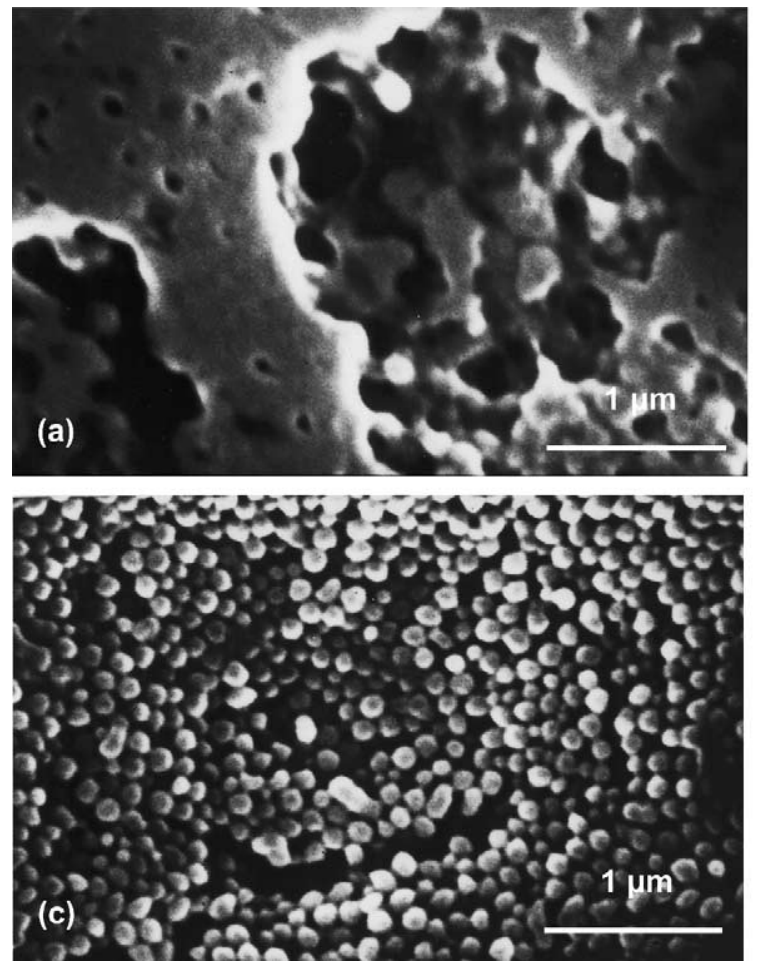

swelling properties at room temperature, therefore the boundary of the particles was indefinite. However, after the second stage reaction, the swelling of the core-shell particles was hindered by the rigid shell, so particles showed distinct boundaries as shown in Fig. 1(b)-(d).

Fig. 2 shows the $\mathrm{COOH}$ groups distribution image of the copolymer particles. The uranyl acetate solution was used to selectively stain anionic (sulfate and carboxylic acid) groups in the particle cross-sections. Under this observation, the anionic functional groups would show a darker image. It could be found that the copolymer particles thus synthesized exhibited a core-shell structure with the stained carboxylic acid groups (MAA) rich in the thin shell region. This result illustrates that the MAA and MMA monomers that were added in the second stage reaction formed the shell region successfully.

\subsection{Swelling behavior}

Because the swelling of the core-shell particle product was hindered by the rigid shell, the swelling ratio and the thermo-responsivity of the core-shell copolymers were largely reduced compared to those of poly(chitosan-coNIPAAm) copolymers prepared in our previous study [29], although the phase transition temperature (LCST) was still about $25-35^{\circ} \mathrm{C}$ as shown in Fig. 3. In these core-shell samples, it was found that chitosan and MAA played more important roles in the swelling behavior of these core-shell particles. Figs. 3 and 4 show the effect of chitosan/NIPAAm
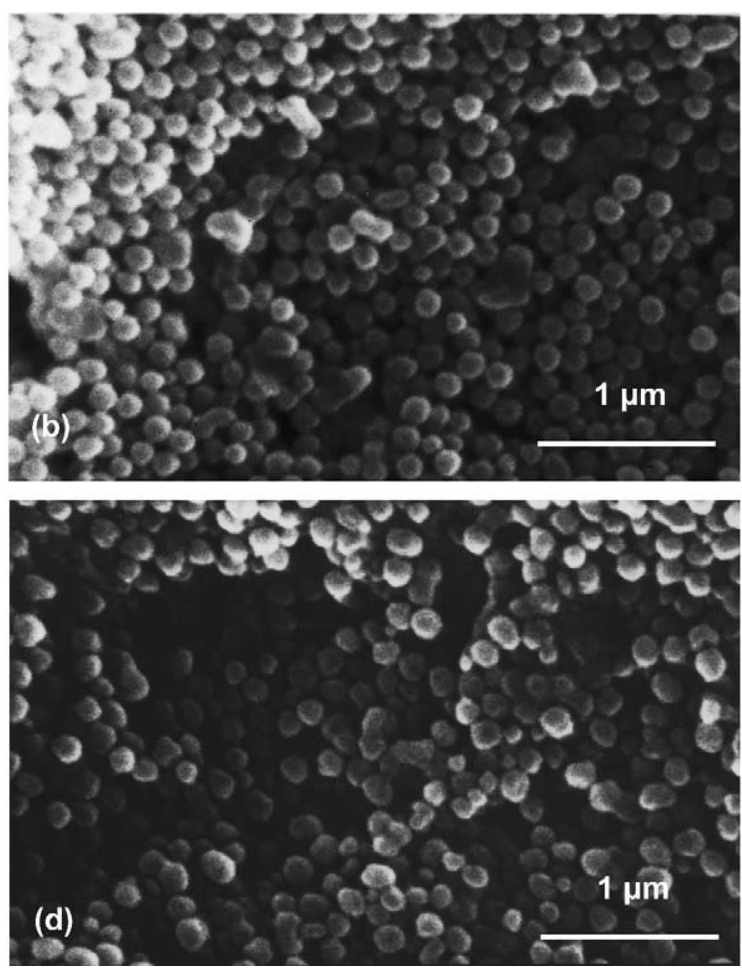

Fig. 1. SEM photographs of copolymer particles (a) 2/10-3\% (core particles without further copolymerization with MAA/MMA) and (b) 2/10-3\%-1/5 (c) 5/10$3 \%-1 / 5$ (d) $2 / 10-5 \%-1 / 5$, (b)-(d) (core-shell particles). 


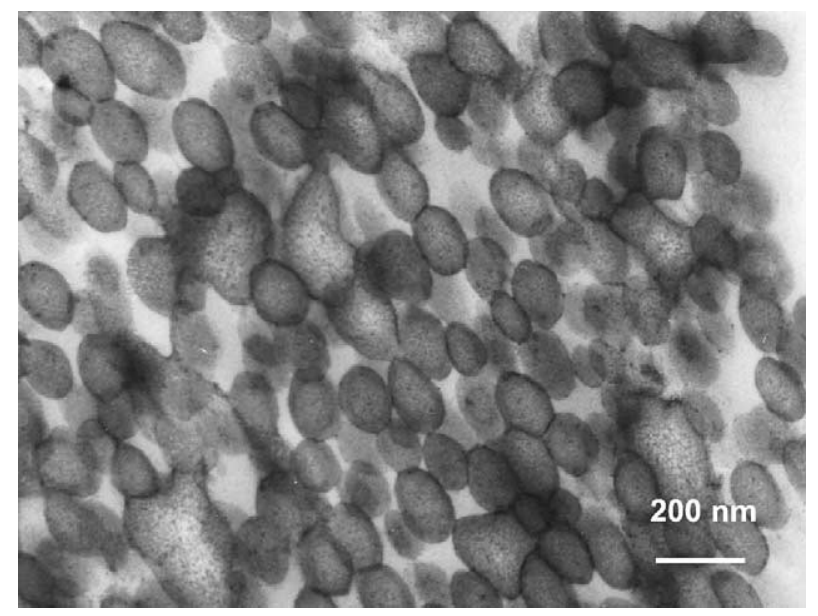

Fig. 2. Transmission electron micrographs of poly(chitosan-co-NIPAAm/MAA-co-MMA) core-shell particles. Anionic sites were selectively stained by using uranyl acetate to appear darker in this image.

(or chitosan/MAA) weight ratio and concentration of crosslinking agent on the swelling ratio of core-shell particles at different temperatures in phosphate buffer solutions with $\mathrm{pH}=4,7.4$, and 9 .
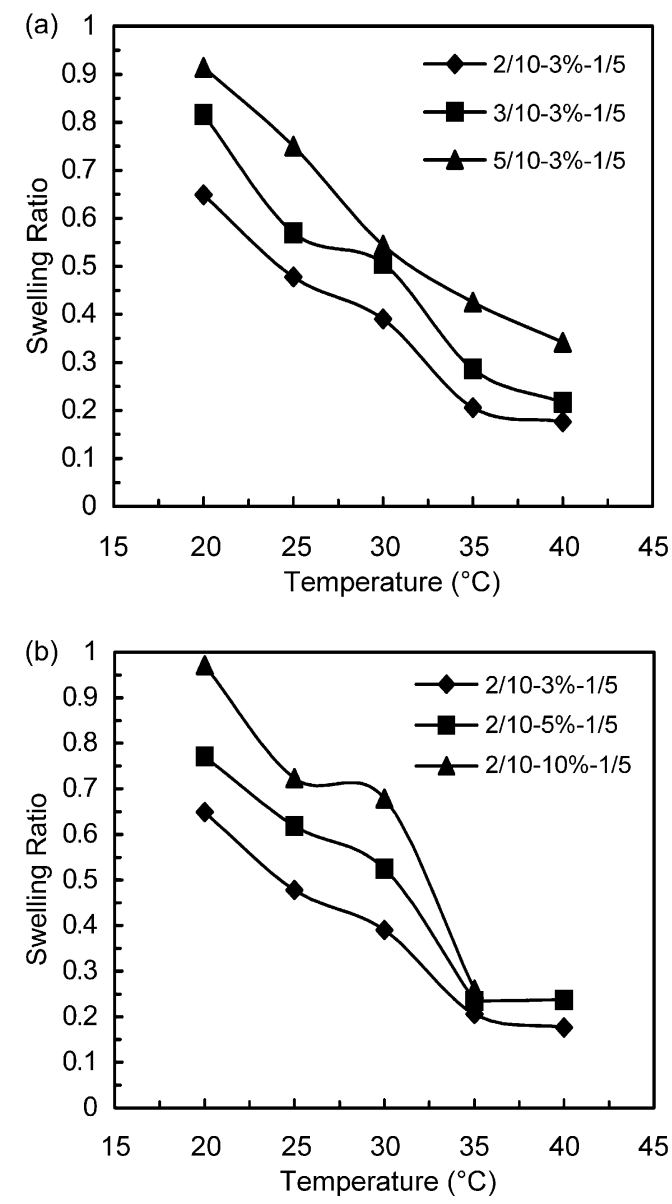

Fig. 3. The swelling ratio of core-shell particles at different temperatures in $\mathrm{pH}=7.4$ phosphate buffer solution: (a) Effect of chitosan/NIPAAm weight ratio (b) effect of the concentration of crosslinking agent.
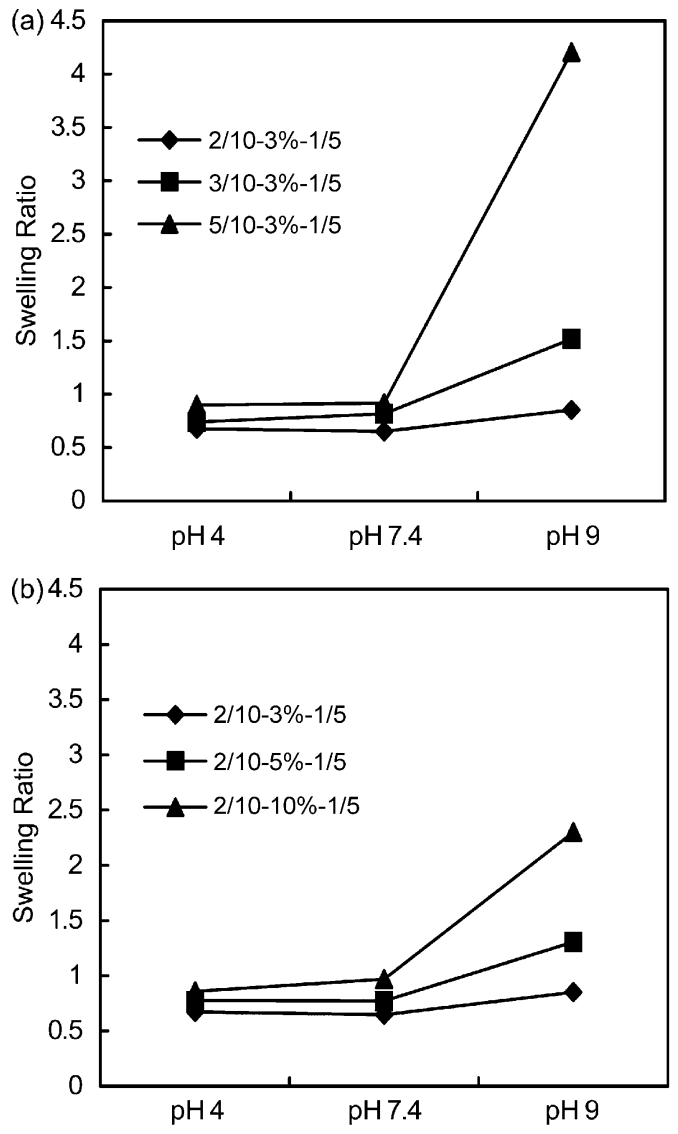

Fig. 4. Swelling ratio of core-shell particles versus $\mathrm{pH}$ value at $20^{\circ} \mathrm{C}$ for samples (a) with different chitosan/NIPAAm weight ratios (b) with different concentrations of crosslinking agent.

\subsubsection{Effect of chitosan/NIPAAm (or chitosan/MAA) weight ratio}

The higher the weight ratio of chitosan/NIPAAm (or chitosan/MAA), the higher the swelling ratio was observed no matter in $\mathrm{pH}=4$ or $\mathrm{pH}=9$ buffer solution. In the $\mathrm{pH}=4$ phosphate buffer solution, the chitosan rich sample had higher swelling ratio because its $\mathrm{NH}_{2}$ groups would ionize to $\mathrm{NH}_{3}^{+}$in this $\mathrm{pH}$ value. In the $\mathrm{pH}=9$ phosphate buffer solution, the chitosan rich sample had higher swelling ratio because the swelling of both MAA and NIPAAm segments in shell and core regions took place. Due to the order of synthesis, poly(chitosan-co-NIPAAm) was in the core region. The swelling of NIPAAm segments was hindered by the rigid shell and the tight crosslinking structure. However, during the shell synthesis process, in the chitosan rich sample, the higher chitosan incorporated in the core enhanced the penetrating of MAA into the core region due to the formation of $\mathrm{COO}^{-} \mathrm{NH}_{3}^{+}$complex. It was sure that more MAA segments could intersperse among poly(chitosan-co-NIPAAm) in the core. So when this sample was put in $\mathrm{pH}=9$ buffer solution, the shell region of particles and the MAA segments existing in the core region were swollen. The boundary constraint in the core, poly(chitosan-co-NIPAAm), was freely released, and then the 
swelling of NIPAAm segments in the core also occurred. Therefore, the swelling ratio of the chitosan rich particles at temperatures lower than LCST of polyNIPAAm was largely increased in the $\mathrm{pH}=9$ phosphate buffer solution as shown in Fig. 4(a).

\subsubsection{Effect of crosslinking agent}

In our previous study [29], the swelling behaviors of poly(chitosan-co-NIPAAm) latex with different crosslinking degree were investigated. It was found that poly (chitosan-coNIPAAm) latex with higher crosslinking degree would have lower swelling ratio. However, when the poly(MAA-coMMA) shell part was added to the poly (chitosan-coNIPAAm) core particle, this trend was reversed as shown in Figs. 3(b) and 4(b). The core-shell particles with higher crosslinking degree had higher swelling ratio either in acidic or basic condition because the higher crosslinking agent would lower the conversion of the first stage reaction and raise the conversion of the second stage reaction. As expected, more chitosan and NIPAAm would copolymerize with monomers, MAA and MMA, to form the shell region when more crosslinking agent was used. The chitosan and NIPAAm incorporated in the shell region enhanced the compatibility between core and shell region and formed channels in the rigid shell to improve thermo-responsive property or the swelling of the core region.

In the $\mathrm{pH}=4$ phosphate buffer solution, the crosslinking agent rich sample would have a higher swelling ratio because more chitosan and NIPAAm channels existed in the shell that caused the swelling of chitosan and NIPAAm segments in both core and shell. But when in the $\mathrm{pH}=9$ phosphate buffer solution, the swelling of MAA and NIPAAm segments dominated the bulk swelling behavior. As the MAA and the NIPAAm segment channels in the shell region were extensively swollen in $\mathrm{pH}=9$ buffer solution, the boundary constraint of the core, was freely released. Therefore, the swelling ratio of the crosslinking agent rich sample at temperatures lower than LCST of polyNIPAAm was significantly increased as shown in Fig. 4(b). However, the increase of swelling ratio of crosslinking agent rich sample was smaller than that of chitosan rich sample when comparing Fig. 4(a) and (b). This was because that the higher crosslinking degree was innately unfavorable for swelling as discussed above.

\subsection{Zeta potential and size of swollen particles}

The swollen size of the latex and the zeta potential of the particles were analyzed in buffer solutions. The zeta potential of particles was between -34 and $-40 \mathrm{mV}$ for all samples when the $\mathrm{pH}$ value was between 7.4 and 9, which was attributed to the carboxylic acid group of MAA in the shell ionized to $\mathrm{COO}^{-}$and the negative charge, $\mathrm{SO}_{4}^{-}$, derived from the initiator. When the buffer solution was changed to $\mathrm{pH}=4$, the combined effects of the positive charge, $\mathrm{NH}_{3}^{+}$, derived from $\mathrm{NH}_{2}$ of chitosan and the negative charge, $\mathrm{SO}_{4}^{-}$, derived from the initiator caused the zeta potential of particles were near $0 \mathrm{mV}$ for all samples.

Fig. 5(a) and (b) show the average diameter of swollen core-shell particles with various weight ratios of chitosan/ NIPAAm or with various concentration of crosslinking agent in buffer solution with different $\mathrm{pH}$ values at $25^{\circ} \mathrm{C}$.

\subsubsection{Effect of chitosan/NIPAAm weight ratio}

The sizes of swollen particles were always larger than those observed by TEM or SEM due to the swelling and/or coagulation effect. The swelling ratio of samples in $\mathrm{pH}=9$ was higher as discussed above, so the average diameter of particles in $\mathrm{pH}=9$ was higher than that in $\mathrm{pH}=7.4$ as expected. However, the average diameter of particles measured in $\mathrm{pH}=4$ were much larger than those in $\mathrm{pH}=$ 7.4 or $\mathrm{pH}=9$ in the light scattering measurement, although the swelling ratio of particles in $\mathrm{pH}=4$ buffer solution was the smallest (Fig. 4). This contradiction was due to the effect of particle coagulation in the light scattering measurement, since particles were unstable with the zeta potential near $0 \mathrm{mV}$ in $\mathrm{pH}=4$ buffer solution as mentioned above.
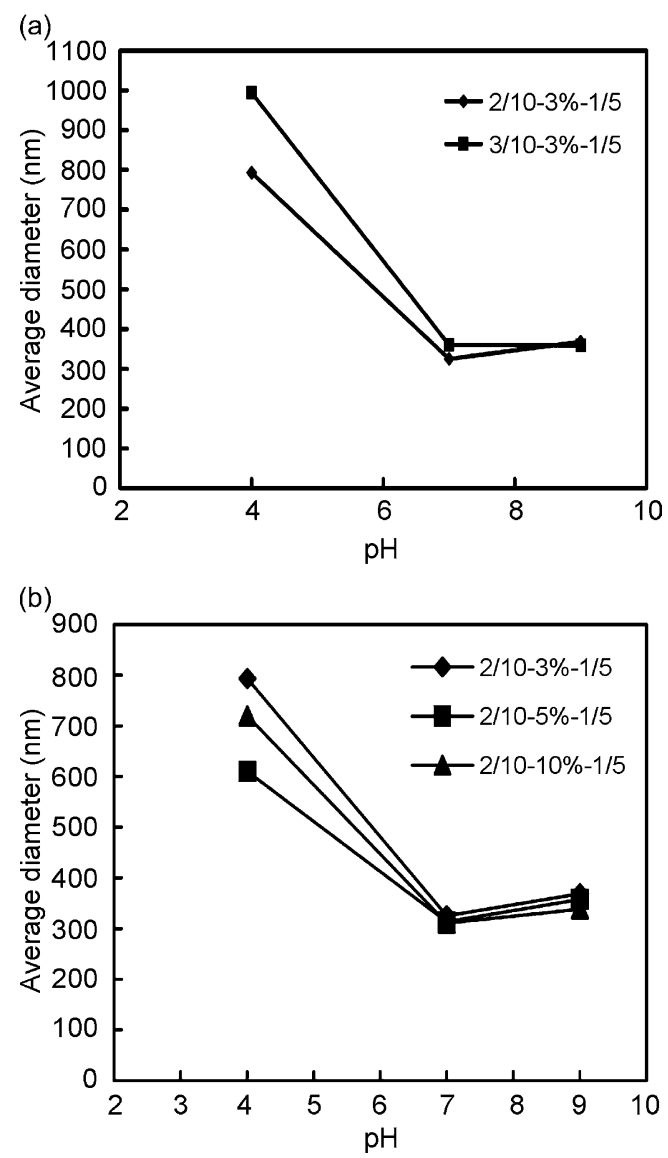

Fig. 5. Average diameter of swollen core-shell particles in buffer solution with different $\mathrm{pH}$ values at $25^{\circ} \mathrm{C}$. (a) effect of weight ratios of chitosan/NIPAAm (b) effect of concentrations of crosslinking agent. 


\subsubsection{Effect of crosslinking agent}

In solutions with $\mathrm{pH}=7.4$ and $\mathrm{pH}=9$, the particles with higher concentration of crosslinking agent had a slightly smaller size. In solution with $\mathrm{pH}=4$, the size of swollen particles was greatly increased due to the combined effect of crosslinking degree, coagulation and swelling of particles. No trend was found in the influence of crosslinking agent on the size of swollen particles.

\subsection{Specific surface area and surface functional group of particles}

In order to know how many carboxylic acid groups were distributed on the core-shell particles as MAA was one of the monomers to synthesize the shell region, the specific surface area and the amount of surface carboxylic acid on the core-shell particles were measured and listed in Table 4.

\subsubsection{Effect of chitosan/NIPAAm weight ratio}

It was expected that the specific surface area would be larger for the chitosan rich sample when the particle size decreased as the weight ratio of chitosan/NIPAAm increased as shown in the TEM observation. However, it was found that the data of specific surface area did not agree with the trend. This was because when samples had a higher swelling ratio, less and larger pores were formed after the freeze-drying process, while the samples with lower swelling ratio would produce more and smaller pores. Considering the pore size effect, when the chitosan/NIPAAm ratio increased, the swelling ratio of samples also increased that caused larger pores and less pore number, so the specific surface area would decrease. As a result, the particle size effect and the pore size effect influenced the specific surface area of particles in opposite ways. From sample $2 / 10-3 \%-1 / 5$ to $3 / 10-3 \%-1 / 5$, the specific surface area increased, but from sample $3 / 10-3 \%-1 / 5$ to $5 / 10-3 \%$ $1 / 5$, the specific surface area decreased. The data of the amount of $\mathrm{COOH}$ on the surface of core-shell particles indicated that the increase of the weight ratio of chitosan/NIPAAm resulted in an increase of $\mathrm{COOH}$ groups per specific surface area of particles.

\subsubsection{Effect of crosslinking agent}

The specific surface area of particles or the amount of $\mathrm{COOH}$ on the particle surface did not show a trend with regard to the concentration variation of crosslinking agent. As discussed above, both particle size effect and pore size effect combined to give the result.

\subsection{Drug releasing estimation}

To evaluate the potential of the core-shell copolymer particles being applied on the drug carrier, caffeine was used as the model drug to carry out the drug-releasing test. In this study, the loading of caffeine into the core-shell latex particles was achieved by the swelling of copolymer particles in the $\mathrm{pH}=7.4$ caffeine containing phosphate buffer solution, thereby the amount of caffeine loaded into the particles was in proportion to the equilibrium swelling ratio shown in Figs. 3 and 4. As expected, the drug might exist in both the particles (loaded caffeine) and the solution (non-loaded caffeine). The drug releasing behaviors of both non-loaded caffeine and loaded caffeine could be observed as seen in Fig. 6.

A reference copolymer tablet formed by blending copolymer particles with drug powders (not by way of swelling) was taken as the comparison. From Fig. 6, it was observed that the blended caffeine was almost completely released from the reference tablet at $37^{\circ} \mathrm{C}$ within $30 \mathrm{~min}$. In other samples loaded with caffeine by swelling process, comparing with the releasing curve of blend sample, the high proportion loss of drug (70-80\%) within 30 min was the amount of non-loaded drug, whereas the very slow releasing curve after $30 \mathrm{~min}$ (near zero release rate) indicated the drug (20-30\%) that was really loaded into the particles (loaded drug) would be trapped and protected from releasing.

From Fig. 6, although the efficiency of drug loading by swelling process was only $20-30 \%$, the drug incorporated in the particles (loaded drug) could be increased by increasing the concentration of drug solution used in the swelling (loading) process. For application, the non-loaded caffeine (about 70-80\%) should be removed from the sample. One way to do this is to dialysis the latex solution. Once the nonloaded drug is removed, the latex particles are expected to have a very slow release rate.

The copolymer particles with higher chitosan/NIPAAm weight ratio or higher concentration of crosslinking agent had a higher swelling ratio, and thus absorbed more amount

Table 4

Specific surface area and $\mathrm{COOH}$ groups of poly(chitosan-co-NIPAAm/MAA-co-MMA) core-shell particles with various chitosan/NIPAAm weight ratios or various concentrations of crosslinking agent

\begin{tabular}{llll}
\hline Sample & $\begin{array}{l}\text { BET specific surface area } \\
\left(\mathrm{m}^{2} / \mathrm{g}\right)\end{array}$ & $\begin{array}{l}\text { COOH group } \times 10^{-3}(\mathrm{~mole} / \\
\mathrm{g})\end{array}$ & COOH group $\times 10^{-4}\left(\mathrm{~mole} / \mathrm{m}^{2}\right)$ \\
\hline $2 / 10-3 \%-1 / 5$ & 17.71 & 2.014 & 1.137 \\
$3 / 10-3 \%-1 / 5$ & 17.78 & 2.056 & 1.156 \\
$5 / 10-3 \%-1 / 5$ & 12.92 & 1.736 & 1.343 \\
$2 / 10-3 \%-1 / 5$ & 17.71 & 2.014 & 1.137 \\
$2 / 10-4 \%-1 / 5$ & 20.08 & 1.944 & 0.968 \\
$2 / 10-5 \%-1 / 5$ & 18.78 & 2.097 & 1.116 \\
\hline
\end{tabular}



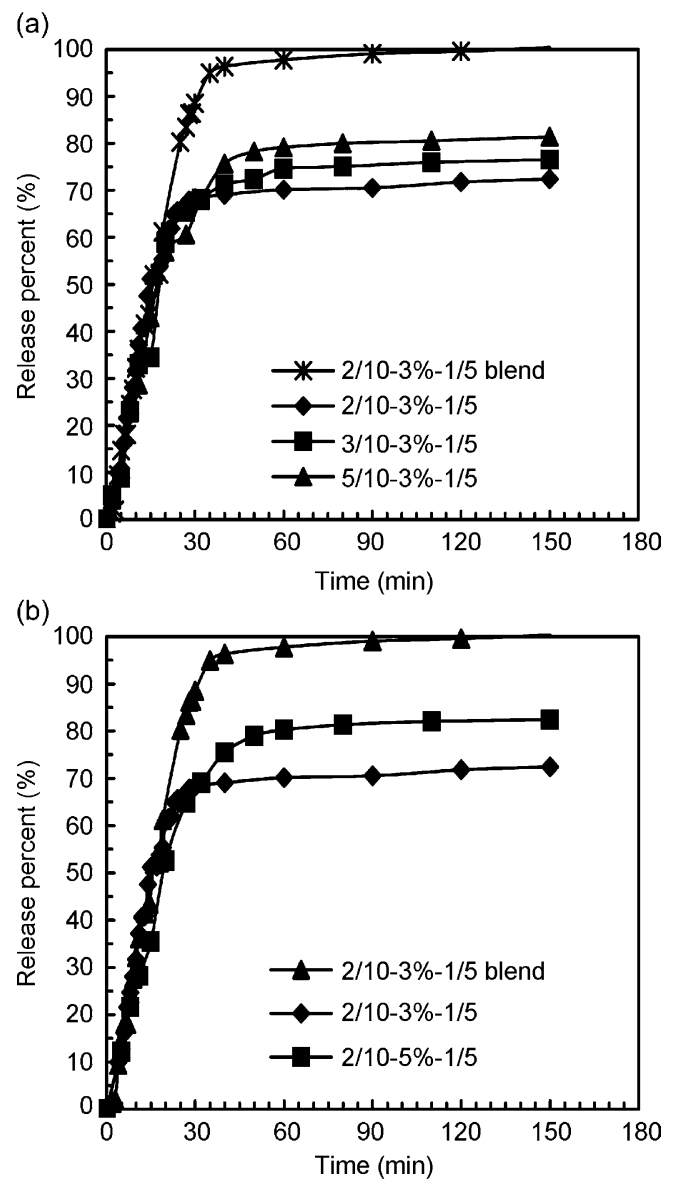

Fig. 6. The percentage of caffeine released from poly(chitosan-coNIPAAm/MAA-co-MMA) copolymer tablets in the $\mathrm{pH}=7.4$ phosphate buffer solution at the temperature of $37^{\circ} \mathrm{C}$. (a) Effect of chitosan/NIPAAm weight ratio (b) effect of concentrations of crosslinking agent.

of caffeine, but the caffeine incorporated in them could not be protected well and tended to diffuse into the solution quickly because of its high swelling behavior. Therefore, it was found in Fig. 6 that the higher the weight ratio of chitosan/NIPAAm or the higher the concentration of crosslinking agent in copolymer particles, the lower the amount of caffeine was kept inside the particles in $\mathrm{pH}=7.4$ buffer solution at $37^{\circ} \mathrm{C}$. In addition, the releasing rate of the chitosan rich or crosslinking agent rich sample was slightly slower in the first 30 min because the caffeine was easily absorbed inside the particles by swelling and then partially released into solution by diffusion.

\subsection{Protein conjugation}

In this work, the bovine serum albumin (BSA) was taken as model protein (ligand) to conjugate on the surface of copolymer particles by the aid of water-soluble carbodiimide (EDC) to evaluate the protein conjugating ability and the potential of the core-shell copolymer particles being applied on the targeting drug carrier. The $\mathrm{COOH}$ groups on the surface of core-shell particles combined with carbodiimide to react with the $\mathrm{NH}_{2}$ group of BSA and then yield the corresponding amide bonding.

Fig. 7 shows the effect of temperature on the amount of BSA conjugated on the surface of core-shell particles. Besides the factor of the concentration of $\mathrm{COOH}$ group on particles, the amount of BSA conjugated on the surface of particles was influenced by another two factors. One was the size of swollen particles and the other was the hydrophobic property of particles. It is well known that the larger swollen particles reduces the steric hindrance of protein (BSA) conjugation, and the greater hydrophobic property of particles increases the adsorption of protein on the surface of particles. Therefore, the favored conditions for protein conjugation were the larger swollen particle size and the greater hydrophobic property of particles the higher concentration of $\mathrm{COOH}$ groups on the particles. So at $37{ }^{\circ} \mathrm{C}$ the amounts of BSA conjugated on particles decreased when the chitosan/NIPAAm weight ratio or concentration of crosslinking agent was increased because the hydrophobic property decreased (Fig. 3), although the carboxylic acid groups per specific surface area of particles varied differently (Table 4). It was also interesting to compare the results of the amounts of BSA conjugated on particles at 37 and at $25^{\circ} \mathrm{C}$. The conjugating amounts were not reduced by the lower reaction rate and the more hydrophilic property of particles at $25^{\circ} \mathrm{C}$, but were increased due to the increase in particle size and the decrease in steric hindrance. Because the swelling was enhanced with the increasing of chitosan/NIPAAm weight ratio or the concentration of crosslinking agent, the enhancement of BAS conjugation at $25^{\circ} \mathrm{C}$ compared to that at $37{ }^{\circ} \mathrm{C}$ was increased as the chitosan/NIPAAm weight ratio or the concentration of crosslinking agent increased. It could be concluded that the thermo-responsive property of these core-shell particles is helpful to enhance the protein conjugation at low temperature and these core-shell

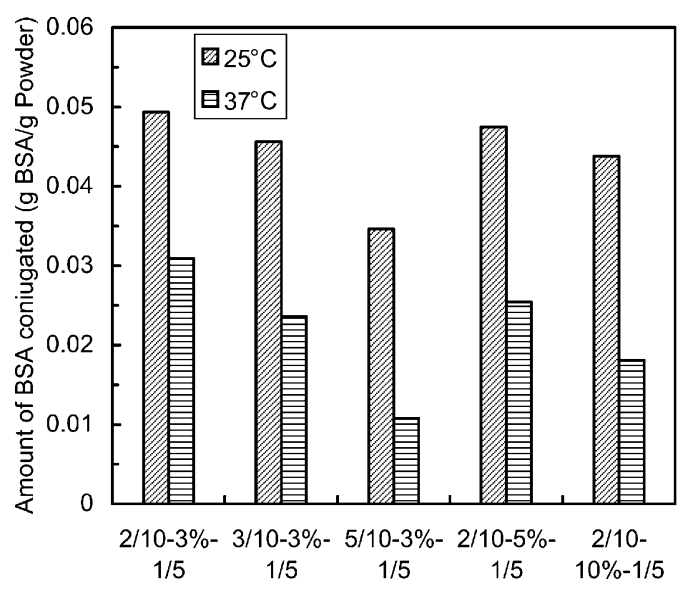

Fig. 7. The effect of temperature on the amount of BSA conjugated on the surface of poly(chitosan-co-NIPAAm/MAA-co-MMA) core-shell particles with various chitosan/NIPAAm weight ratios or various concentrations of crosslinking agent. 
particles have the potential for targeting drug carrier application.

\section{Conclusion}

The crosslinked poly(chitosan-co-NIPAAm/MAA-coMMA) thermal-sensitive and core-shell copolymer particles were successfully synthesized in this work. The relationships between the conversion, swelling, zeta potential, average diameter, specific surface area of particles, drug releasing and protein conjugation were studied. With an increase of the weight ratio of chitosan/NIPAam, the size of core-shell particles decreased, and the swelling ratio of the sample increased. With an increase of the concentration of crosslinking agent, the size of core-shell particles remained almost the same, and the swelling ratio of the sample increased. The swelling behavior and the zeta potential of particles were dominated by the chitosan in the $\mathrm{pH}=4$ buffer solution but it was dominated by the MAA in the $\mathrm{pH}=9$ buffer solution. In the drug releasing estimation, 20$30 \mathrm{wt} \%$ of caffeine could be loaded inside the copolymer particles by the swelling process. And the loaded caffeine was protected from releasing effectively at $37{ }^{\circ} \mathrm{C}$. In the protein conjugation, the amount of BSA conjugated on the surface of particles was mainly influenced by the size of swollen particles or the hydrophobic property of particles. In this study, the conjugation amounts at either 37 or $25^{\circ} \mathrm{C}$ were decreased with increasing the weight ratio of chitosan/NIPAAm or the concentration of crosslinking agent. The conjugation amounts increased significantly at $25^{\circ} \mathrm{C}$ because of the increase in swollen particle size and the decrease in steric hindrance.

\section{References}

[1] Heskins M, Guillet JE, James E. J Macromol Sci Chem 1968;8:1441.
[2] Bae YH, Okano T, Kim SW. J Polym Sci B, Polym Phys 1990;28(6): 923-36.

[3] Binkert T, Oberreich J, Meewes M, Nyffenegger R, Ricka J. Macromolecules 1991;24(21):5806-10.

[4] Ilavsky M. Macromolecules 1982;15(3):782-8.

[5] Ohmine I, Tanaka T. J Chem Phys 1982;77(11):5725-9.

[6] Park TG. Biomaterials 1999;20(6):517-21.

[7] Zhang X, Wu D, Chu CC. Biomaterials 2004;25(19):4719-30.

[8] Huglin MB, Liu Y, Velada JL. Polymer 1997;38(23):5785-91.

[9] Yoo MK, Sung YK, Lee YM, Cho CS. Polymer 2000;41(15):5713-9.

[10] Neradovic D, Soga O, Van Nostrum CF, Hennink WE. Biomaterials 2004;25(12):2409-18.

[11] Kitano H, Yan C, Nakamura K. Makromol Chem 1991;192(12): 2915-23.

[12] Kawaguchi H, Fujimoto K, Muzuhara Y. Colloid Polym Sci 1992; 270(1):53-7.

[13] Snowdon MJ. J Chem Soc Chem Commun 1992;(11):803.

[14] Snowdon MJ, Vincent B. J Chem Soc Chem Commun 1992;(16): $1103-4$

[15] Kiminta D, Costello B, Lenon S, Luckham PF. Mater Res Soc Symp Proc 1993;289:13-18.

[16] Makino KS, Yamamoto K, Fujimoto H, Kawaguchi H, Ohshima H. J Colloid Interface Sci 1994;166(1):251-8.

[17] Zhu PW, Napper DH. J Colloid Interface Sci 1994;164(2):489-94.

[18] Zhu PW, Napper DH. J Colloid Interface Sci 1994;168(2):380-5.

[19] Lowe TL, Tenhu H, Tylli H. J Appl Polym Sci 1999;73(16):1031-9.

[20] Kidchob T, Kimura S, Imanishi Y. J Controlled Release 1998;50(13):205-14.

[21] Chung JE, Yokoyama M, Yamato M, Aoyagi T, Sakurai Y, Okano T. J Controlled Release 1999;62(1-2):115-27.

[22] Zhang K, Wu XY. Biomaterials 2004;25(22):5281-91.

[23] Roos A, Klee D, Schuermann K, Höcker H. Biomaterials 2004; 24(24):4417-23.

[24] Kono K, Henmi A, Yamashita H, Hayashi H, Takagishi T. J Controlled Release 1999;59(1):63-75.

[25] Wang M, Qiang J, Fang Y, Hu D, Cui Y, Fu X. J Polym Sci A, Polym Chem 2000;38(3):474-81.

[26] Kim SY, Cho SM, Lee YM, Kim SJ. J Appl Polym Sci 2000;78(7): 1381-91.

[27] Wang M, Fang Y, Hu D. React Funct Polym 2001;48(1-3):215-21.

[28] Lee CF, Wen CJ, Chiu WY. J Polym Sci A, Polym Chem 2003; 41(13):2053-63.

[29] Lee CF, Wen CJ, Lin CL, Chiu WY. J Polym Sci A, Polym Chem 2004;42(12):3029-37.

[30] Saunders BR, Vincent B. Adv Colloid Interface Sci 1999;80(1):1-25.

[31] Hsu SC, Don TM, Chiu WY. Polym Degrad Stab 2002;75(1):73-83. 